\title{
A cross-sectional investigation into the occupational and socio- demographic characteristics of British police force employees reporting a dietary pattern associated with cardiometabolic risk: findings from the Airwave Health Monitoring Study
}

\author{
Rachel Gibson ${ }^{1}$ - Rebeca Eriksen ${ }^{1} \cdot$ Deepa Singh $^{2} \cdot$ Anne-Claire Vergnaud $^{2} \cdot$ \\ Andrew Heard ${ }^{2}\left(\right.$ ) $\cdot$ Queenie Chan $^{2}\left(\mathbb{D} \cdot\right.$ Paul Elliott $^{2} \cdot$ Gary Frost $^{1}$
}

Received: 10 April 2017 / Accepted: 13 October 2017 / Published online: 2 November 2017

(C) The Author(s) 2017. This article is an open access publication

\begin{abstract}
Purpose The aims of this study were to (1) determine the association between diet quality using the Dietary Approaches to Stop Hypertension (DASH) score and cardiometabolic risk in a British working population and (2) identify employee characteristics associated with reporting a poorer quality dietary pattern.

Methods British police employees enrolled (2007-2012) into the Airwave Health Monitoring Study $(n=5527)$ were included for sex-specific cross-sectional analyses. Dietary intakes were measured using 7-day food records. DASH score was calculated to determine diet quality. Logistic regression evaluated associations between (1) diet quality and increased cardiometabolic risk (defined as $\geq 3$ risk markers: dyslipidaemia, elevated blood pressure, waist circumference, CRP or HbA1c), and (2) poor diet quality (lowest fifth of DASH score distribution) and employee characteristics.

Results Employees recording a poor diet quality had greater odds (OR) of increased cardiometabolic risk independent of established risk factors (demographic, lifestyle and occupational) and BMI: men OR 1.50 (95\% CI
\end{abstract}

Electronic supplementary material The online version of this article (doi:10.1007/s00394-017-1562-4) contains supplementary material, which is available to authorized users.

Rachel Gibson

Rachel.gibson13@imperial.ac.uk

1 Nutrition and Dietetic Research Group, Faculty of Medicine, Imperial College, University of London, London W12 0NN, UK

2 Department of Epidemiology and Biostatistics, MRC-PHE Centre for Environment and Health, School of Public Health, Imperial College, London W2 1PG, UK
1.12-2.00), women: OR 1.84 (95\% CI 1.19-2.97) compared to the healthiest diet group. Characteristics associated with reporting a poor quality diet were employment in Scotland vs. England: men OR 1.88 (95\% CI 1.53-2.32), women: OR 1.49 (95\% CI 1.11-2.00), longer working hours ( $\geq 49$ vs. $\leq 40$ h) men: OR 1.53 (95\% CI 1.21-1.92) women: OR 1.53 (95\% CI 1.12-2.09). For men, job strain (high vs. low) was associated with reporting a poor diet quality OR 1.66 (95\% CI 1.30-2.12).

Conclusions The general population disparities in diet quality between England and Scotland were reflected in British police employees. The association of longer working hours and job strain with diet quality supports the targeting of workplace nutritional interventions.

Keywords DASH (Dietary Approaches to Stop Hypertension Score) $\cdot$ Cardiometabolic risk $\cdot$ Police $\cdot$ Diet

\section{Introduction}

Employment is considered beneficial to health and wellbeing when occupational hazards are controlled [1]. Two types of work-related disease have been described by the World Health Organization: (1) 'occupational diseases' which occur as a direct result of workplace-specific exposures and (2) 'multifactorial diseases' which describe diseases that occur in the general population, but may be exacerbated or partially caused by workplace exposures [1]. The last few decades have produced an increasing number of studies suggesting that type two diabetes (T2DM) and cardiovascular disease (CVD) are multifactorial work-related diseases. Examples of occupational exposures associated with a disproportionate risk of poor 
cardiometabolic health include shift work [2], long working hours [3], and job strain [4].

It has been estimated that about a third of daily calorie intake is consumed in the workplace [5]. Therefore, diet should be an important consideration in the relationship between workplace exposures with cardiometabolic disease risk. The role of diet in the aetiology of cardiometabolic disease development and mortality is well established [6], with dietary modification central to the primary prevention and management of CVD and T2DM $[7,8]$.

The two foremost food-based dietary patterns that have shown to be beneficial in the management of cardiometabolic disease risk are the Mediterranean diet and the dietary approaches to stop hypertension diet (DASH) [9]. Dietary scores have been developed to measure dietary intake against these two dietary patterns in large cohort studies. Although both scores estimate a similar cardiometabolic disease risk reduction, it has been suggested that DASH captures the dietary characteristics related to T2DM to a greater extent than other diet quality scores [10]. Additionally, a meta-analysis of 20 randomised controlled trials of the DASH diet in the management metabolic risk found that in addition to a significant lowering of blood pressure, the DASH diet showed positive effects in the lowering of LDL cholesterol [11]. The DASH dietary pattern is characterised by high fruit, vegetable, low fat dairy and wholegrain intakes with low red meat, sugarsweetened beverage and salt intakes [12].

To manage the increasing burden of cardiometabolic disease, public health strategies need to take account of wider environmental exposures as well as individual health behaviours. There is, therefore, a growing need to understand the relative influence of factors within different environments, such as the workplace, on dietary choice [13]. Research examining the relationship between occupational exposures and diet is currently lacking in large occupational groups in the UK.

The British police force employs over 250000 men and women [14] across a range of job roles and geographical regions with varying working hour arrangements; however to date the diet, occupational, lifestyle and the cardiometabolic health of this large occupational group in the UK has not been studied. The Airwave Health Monitoring Study is a longitudinal study of British police force employees launched in 2004, with 42,112 participants enrolled at the end of 2012 [15]. Since April 2007, a sub-sample of participants completed a 7-day estimated weighted food diary $(n=15,404)$. The aims of this paper are to (1) measure the sex-specific association between diet quality determined Dietary Approaches to Stop Hypertension (DASH) score and cardiometabolic risk and (2) identify employee characteristics associated with reporting a dietary pattern associated with elevated cardiometabolic disease risk.

\section{Subjects and methods}

\section{Participants}

We conducted a cross-sectional study using baseline data collected as part of the Airwave Health Monitoring Study between 2007 and 2012. The Airwave Health Monitoring Study was open to all police forces in Great Britain, recruitment procedures and baseline characteristics have been described previously [15]. For the purpose of the present study, we included participants who had baseline dietary data available at the end of December 2015. We excluded participants with self-reported chronic disease diagnosis: angina, heart disease, angina, chronic obstructive pulmonary disease, cancer, chronic liver disease, thyroid disease and/or previous stroke $(n=322)$ as these diseases may affect cardiometabolic markers of interest. The present study sample $(n=5527)$ was comparable across key characteristics the total study sample $(n=42,112)$ [15]. The Airwave Health Monitoring Study is approved by the National Health Service Multi-site Research Ethics Committee (MREC/13/ NW/0588).

\section{Assessment of dietary intake}

Dietary intake was measured using 7-day estimated weighted food diaries. Participants were provided with written instructions and requested to provide details on cooking methods, brand names and portion sizes. To aid portion size estimation, photographs based on those developed by Nelson et al. were provided [16]. Calculation of nutritional intake was conducted using Dietplan6.7 software (Forestfield Software Ltd, Horsham, UK) which was based on the McCance and Widdowson's 6th Edition Composition of Foods UK Nutritional Dataset (UKN). A team of trained coders 'coded' the diaries (matching of food and drink items recorded to a UKN database code and a portion size) follwing a study-specific standard protocol [17]. All coders were qualified nutritionists, dietitians or working towards a nutrition qualification. Mean energy intake ( $\mathrm{kcal} /$ day) was derived from total energy intake divided by the number of complete food diary days. Energy-adjusted intakes (percentage contribution to total energy intake) were calculated for key macronutrient (total carbohydrate, non-milk extrinsic sugars, total fat, saturated fat, protein and alcohol). The Goldberg method was applied to estimate prevalence of energy intake misreporting [18]. The methodology applied and the results of estimated energy misreporting in this cohort have previously been reported in detail [17]. In the present study, we conducted sensitivity analyses to explore if energy intake misreporting among the Airwave Health Monitoring Study participants was a potential source of bias in the relationship between diet quality and cardiometabolic risk. 


\section{Diet quality measurement}

Mean daily intakes were calculated for 'positive' and 'negative' food groups (g/day) and sodium ( $\mathrm{mg} /$ day) for each participant (positive food groups: Whole grains, low fat dairy, total fruit, vegetables excluding white potatoes, nuts, legumes and seeds; negative food groups: sugar-sweetened beverages, processed red meat and sodium) Supplementary Table S1 details the food group descriptions. The DASH scores were calculated as described by Fung et al. [12]. Participants were stratified by sex and the quintile (Q) of intake for each food group calculated using the following quintile score system. For positive food groups: $\mathrm{Q} 1=1$ point, $\mathrm{Q} 2=2$ points, $\mathrm{Q} 3=3$ points, $\mathrm{Q} 4=4$ points, $\mathrm{Q} 5=5$ points and for negative food groups reverse scoring was applied: $\mathrm{Q} 1=5$ points, $\mathrm{Q} 2=4$ points, $\mathrm{Q} 3=3$ points, $\mathrm{Q} 4=2$ points, $\mathrm{Q} 5=1$ point. The score ranges from 8 to 40 , with a higher score thus corresponds to a more healthy diet pattern. Supplementary Table S2 shows the quintile cut-offs used to calculate the DASH score for the present study.

\section{Anthropometric, blood pressure and biochemical measurements}

Enrolled participants attended a regional health-screening clinic. Trained research nurses used a standard protocol to conduct all clinical examinations as described previously [15]. Briefly, venous blood samples were drawn in the nonfasted state. Samples were processed on site and then transported (stored in a thermoporter at $0-4{ }^{\circ} \mathrm{C}$ ) for processing at a designated study laboratory. Assays (HDL, non-HDL cholesterol, high sensitivity-C-reactive protein and $\mathrm{HbA1c}$ ) were performed using serum, with the exception of $\mathrm{HbA1c}$, which used whole EDTA blood samples (IL 650 analyser Instrumentation Laboratory, Bedford, Massachusetts, USA). Sitting blood pressure was measured using the Omron HEM 705-CP digital BP monitor (Omron Health Care). Three measurements were taken $30 \mathrm{~s}$ apart and the average recorded. Body weight was measured to the nearest $0.05 \mathrm{~kg}$ using digital scales (Marsden digital weighing scale). Standing height was measured to the nearest $0.1 \mathrm{~cm}$ (Marsden $\mathrm{H} 226$ portable stadiometer). Body mass index (BMI) was calculated as weight $(\mathrm{kg}) /$ weight $\left(\mathrm{m}^{2}\right)$. Waist circumference was measured between the lower rib margin and the iliac crest in the mid-axillary line using a Wessex-finger/joint measure tape (Seca 201, Seca).

\section{Definition of cardiometabolic risk}

For each participant, cardiometabolic risk was estimated based on the presence (yes/no) of five established risk parameters: (1) central adiposity (waist circumference $\geq 94 \mathrm{~cm}$ for men and $\geq 80 \mathrm{~cm}$ for women), (2) dyslipidaemia (HDL of $<1.0 \mathrm{mmol} / \mathrm{L}$ for men or $<1.3 \mathrm{mmol} / \mathrm{L}$ for women, and/or non-HDL $\geq 4.0 \mathrm{mmol} / \mathrm{L}$, and/or prescribed lipid lowering medication), (3) elevated blood pressure (systolic $\geq 130 \mathrm{mmHg}$ and/or diastolic $\geq 85 \mathrm{mmHg}$, and/or prescribed hypotensive medication), (4) inflammation (Hs$\mathrm{CRP} \geq 3 \mathrm{mg} / \mathrm{L}<10 \mathrm{mg} / \mathrm{L}$ ) and (5) impaired blood glucose control (HbA1c $\geq 5.7 \%$ and/or prescribed medication for glucose control). Increased cardiometabolic risk was defined as having three or more of these risk factors. Medication descriptions were matched to British National Formulary category and clinical indication determined.

\section{Employee characteristics}

Information on occupational, lifestyle, medical history, socioeconomic and, demographic factors was collected during the health-screen visit using a structured on-line questionnaire. Total working hours (including usual weekly overtime) was classified into categories $(<40,41$ to $48, \geq 49 \mathrm{~h}$ per week) based on previous research [3]. Job descriptors were collapsed from 31 to 2 categories of "work environment' based on predominant working environment (mobile, or office based). Job strain dimensions were measured using a four-point Likert scale in response to six job content statements derived from the Karosek Job Content Questionnaire [19]. The quadrant approach was used to define job-strain [20]: high (low control, high demand), active (high control, high demand), passive (low, control, low demand), and low strain (high control, low demand). Physical activity information was collected using The International Physical Activity Questionnaire Short Form (IPAQ-SF) [21] which calculates metabolic equivalent (MET) minutes per week across three exercise domains (walking, moderate and vigorous) with participants categorised as undertaking a high, moderate or low level of activity [22]. Weekly TV viewing time was recorded as part of the lifestyle questionnaire in multiples of $15 \mathrm{~min}$, and hours sitting per weekday were recorded in 5-h intervals. These variables were categorised into three groups (high, moderate and low) based on tertile cut-off values (TV viewing hours per week: low $<6$, moderate 6 to 15 , high $>15$; weekly hours sitting on weekdays: low $<20$, moderate 20 to 40 , high $>40$ ).

\section{Statistical analysis}

All statistical analyses were undertaken using SAS version 9.3 (SAS Institute, Cary, NC, USA). To assess differences between two groups, independent $t$ tests were used for data with a normal distribution (mean and standard deviation presented) and Mann-Whitney $U$ tests were used otherwise (median and inter quartile range presented). Associations across categorical variables were analysed using ChiSquared test $\left(\chi^{2}\right)$ with post-hoc comparisons conducted to 
determine the difference between subgroups [23]. To correct for multiple comparisons, the Bonferroni method was applied. As there were significant differences in occupational and lifestyle factors between men and women, we stratified all further analyses by sex. We used logistic regression to estimate the odds of having three or more markers of cardiometabolic risk per quintile of DASH score. The linear contrast function (SAS software) was used to test the presence of a linear trend $\left(P_{\text {trend }}\right)$ across the five ascending categories of DASH score (based on quintile ranges presented in Supplementary Table 3 ) and the association of having three or more cardiometabolic risk markers. Orthogonal polynomial coefficients (SAS function: PROC IML) were generated and applied to the contrast statement to correct for the unequal spacing between median values of each quintile of DASH score. Established confounding variables were included in the two models: (1) minimally adjusted: age (continuous), (2) fully adjusted: age plus categorical variables: physical activity, smoking status, education, TV viewing, job strain, menopause status (women only) and continuous variables: mean energy intake and mean alcohol g/day. Where a high level of collinearity was indicated between confounding variables (hours sitting and TV viewing, working hours and job strain) the variable explaining the greatest amount of variability was retained in the model. Due to the level of missing data for employment rank and work environment, these variables were not included in the multivariable analyses to maintain analytical sample size. Body mass index (BMI) was included in a separate model (continuous variable) as it potentially lies on the causal pathway between diet and markers of cardiometabolic health. Second, we used logistic regression to investigate the employee characteristics associated with consuming a poor quality diet (classified in the lowest DASH score group). Initially, we conducted bivariate logistic regression to determine the relationship between poor diet quality (yes, no) with each covariate. We then adjusted for established predictors of diet quality based on previous studies (age, smoking status, household income, physical activity, marital status, education, TV viewing, and country). Odds ratios (OR) are presented with corresponding $95 \%$ confidence intervals $(95 \% \mathrm{CI})$.

\section{Results}

\section{Population characteristics}

The characteristics of the population sample by sex are shown in Table 1. From the 5527 participants included in the present study $60.3 \%$ were male, men were significantly older than women: $42.4 \pm 8.9$ vs. $39.5 \pm 9.5$ years, $p<0.0001$. There were significant differences between men and women for all occupational characteristics measured, with men more likely to be in higher rank ( 8.8 vs. $2.6 \%, p<0.0001$ ), and have mobile job roles (58.7 vs. $45.1 \%, p<0.0001$ ) and work $49 \mathrm{~h}$ or more per week $(26.2$ vs. $12.5 \%, p<0.0001)$. Women were more likely to be classified as having no markers of cardiometabolic risk compared to men (9.1 vs. $18.0 \%)$, while men were more likely to be classified as having three or more elevated risk markers (41.4 vs. $24.8 \%$ ). Of those participants that had three or more risk factors, $84 \%$ men and $95 \%$ women, had central adiposity as one of the risk factors. Waist circumference was strongly correlated with BMI for men and women (Partial Pearson Correlation, age adjusted: men $r=0.84, p<0.0001$, women $r=0.88, p<0.0001$ ).

\section{Dietary descriptive statistics}

There were significant differences between sources of energy intake between men and women. Women derived more energy from carbohydrates $(48.0 \pm 7.1$ vs. $46.7 \pm 6.9 \%$, $p<0.0001)$ and non-milk extrinsic sugars $(12.3 \pm 5.2 \mathrm{vs}$. $11.6 \pm 4.9 \%, p<0.0001)$, while men obtained more energy from alcohol (4.4 IQR 7.6 vs. 2.9 IQR 6.6\%, $p<0.0001$ ). With the exception of whole grains and sodium, there were significant differences between energy-adjusted intakes across all food groups and dietary fibre, Table 2. Women consumed more, low fat dairy per $1000 \mathrm{kcal}$ than men, while men consumed more red meat (including processed meat) per $1000 \mathrm{kcal}$. Women consumed diets with a higher concentration of fruit, vegetables, legumes, dairy and sugarsweetened beverages $(\mathrm{g} / 1000 \mathrm{kcal})$ compared to men.

\section{DASH score and cardiometabolic risk}

Logistic regression analyses showed a dose-response relationship across quintiles of DASH score and the odds of having three or more markers of cardiometabolic risk for men and women ( $p$ trend $<0.0001$ ), Table 3 . The association remained for men and women in the lowest DASH score group with an OR of 1.84 (95\% CI 1.43, 2.38) and 1.92 (95\% CI $1.35,2.84)$, respectively, for recording three or more cardiometabolic risk factors. There was an attenuation of the OR following further adjustment for BMI (men: OR 1.50, 95\% CI 1.12, 2.00; women: OR 1.84, 95\% CI 1.19, 2.97), however, the significant dose-response relationship across quintile groups of DASH score and three or more metabolic risk factors were maintained.

\section{Predictors of poor quality diet pattern}

In bivariate logistic regression household income was associated with poor diet quality (lowest fifth of DASH score distribution) in men but not in women, though in men the negative association observed in the highest income brackets did not remain significant after 
Table 1 Comparison of demographic, lifestyle and occupational characteristics across men and women in the Airwave Health Monitoring Study cohort ( $n$ 5527)

\begin{tabular}{|c|c|c|c|c|c|}
\hline \multirow[b]{2}{*}{$N, \%$} & \multicolumn{2}{|c|}{ Women } & \multicolumn{2}{|l|}{ Men } & \multirow[t]{2}{*}{$P^{*}$} \\
\hline & 2195 & 39.7 & 3332 & 60.3 & \\
\hline Age, years, mean SD & 39.5 & 9.5 & 42.4 & 8.9 & $<0.0001$ \\
\hline \multicolumn{6}{|l|}{$N, \%$} \\
\hline White & 2153 & 98.1 & 3222 & 96.8 & \\
\hline Relationship status & & & & & $<0.0001$ \\
\hline Cohabiting & 451 & 21.3 & 464 & 14.1 & \\
\hline Married & 216 & 10.2 & 219 & 6.7 & \\
\hline Single & 1046 & 49.5 & 2375 & 72.2 & \\
\hline Divorced/separated & 401 & 19.0 & 230 & 7.0 & \\
\hline Missing & 81 & & 44 & & \\
\hline Education & & & & & 0.006 \\
\hline Left school before taking GCSE & 74 & 3.4 & 155 & 4.7 & \\
\hline GCSE or equivalent & 611 & 27.8 & 1023 & 30.7 & \\
\hline Vocational qualifications & 160 & 7.3 & 240 & 7.2 & \\
\hline A levels / Highers or equivalent & 718 & 32.7 & 1079 & 32.4 & \\
\hline Bachelor Degree or equivalent & 477 & 21.7 & 637 & 19.1 & \\
\hline Postgraduate qualifications & 155 & 7.1 & 197 & 5.9 & \\
\hline Annual household income & & & & & $<0.0001$ \\
\hline Less than $£ 32,000$ & 580 & 26.4 & 298 & 8.9 & \\
\hline$£ 32,000-£ 47,999$ & 246 & 11.2 & 390 & 11.7 & \\
\hline$£ 48,000-£ 57,999$ & 698 & 31.8 & 1470 & 44.1 & \\
\hline$£ 58,000-£ 77,999$ & 428 & 19.5 & 849 & 25.5 & \\
\hline More than $£ 78,000$ & 243 & 11.1 & 324 & 9.7 & \\
\hline Employment (force) country & & & & & $<0.0001$ \\
\hline England & 1629 & 74.5 & 2299 & 69.2 & \\
\hline Scotland & 310 & 14.2 & 647 & 19.5 & \\
\hline Wales & 248 & 11.3 & 377 & 11.4 & \\
\hline Missing & 8 & & 9 & & \\
\hline Rank & & & & & $<0.0001$ \\
\hline Non-ranked police staff/other & 1099 & 57.2 & 612 & 21.0 & \\
\hline Police Constable/ Sergeant & 771 & 40.1 & 1983 & 68.2 & \\
\hline Inspector/Chief Inspector or above & 50 & 2.6 & 255 & 8.8 & \\
\hline Other & 3 & 0.2 & 59 & 2.0 & \\
\hline Missing & 272 & 12.0 & 423 & 12.7 & \\
\hline Work environment & & & & & $<0.0001$ \\
\hline Mainly office duties & 689 & 54.9 & 998 & 41.3 & \\
\hline Mainly mobile duties & 566 & 45.1 & 1417 & 58.7 & \\
\hline Missing/unclassified & 940 & 42.8 & 917 & 27.5 & \\
\hline Total hours worked per week & & & & & $<0.0001$ \\
\hline$\leq 40 \mathrm{~h}$ & 1387 & 63.2 & 1197 & 35.9 & \\
\hline $41-48 \mathrm{~h}$ & 533 & 24.3 & 1263 & 37.9 & \\
\hline$\geq 49$ & 275 & 12.5 & 872 & 26.2 & \\
\hline Job Strain & & & & & $<0.0001$ \\
\hline Low (high control, low demand) & 570 & 26.0 & 1079 & 32.4 & \\
\hline Passive (low control, low demand) & 555 & 25.3 & 585 & 17.6 & \\
\hline Active (high demand, high control) & 538 & 24.5 & 952 & 28.6 & \\
\hline High (high demand, low control) $(\mathrm{N}, \%)$ & 532 & 24.2 & 716 & 21.5 & \\
\hline Physical activity $\dagger$ & & & & & $<0.0001$ \\
\hline Low & 314 & 14.3 & 346 & 10.4 & \\
\hline Moderate & 1061 & 48.3 & 1455 & 43.7 & \\
\hline High & 820 & 37.4 & 1531 & 45.9 & \\
\hline
\end{tabular}


Table 1 (continued)

\begin{tabular}{|c|c|c|c|c|c|}
\hline & Women & & Men & & $P^{*}$ \\
\hline Smoking status & & & & & $<0.0001$ \\
\hline Never smoker & 1489 & 68.1 & 2333 & 70.3 & \\
\hline Former smoker & 472 & 21.6 & 753 & 22.7 & \\
\hline Current smoker & 227 & 10.4 & 234 & 7.1 & \\
\hline Missing & 17 & & 2 & & \\
\hline Sleep & & & & & $<0.0001$ \\
\hline $5 \mathrm{~h}$ or less & 140 & 6.4 & 165 & 5.0 & \\
\hline $6 \mathrm{~h}$ & 471 & 21.5 & 1005 & 30.2 & \\
\hline $7 \mathrm{~h}$ & 903 & 41.1 & 1448 & 43.5 & \\
\hline $8 \mathrm{~h}$ & 586 & 26.7 & 624 & 18.7 & \\
\hline $9 \mathrm{~h}$ or more & 95 & 4.3 & 89 & 2.7 & \\
\hline Missing & & & 1 & & \\
\hline Sitting (total weekdays) & & & & & 0.09 \\
\hline Low $(<20 \mathrm{~h})$ & 707 & 32.2 & 1023 & 30.7 & \\
\hline Moderate $(20-40 \mathrm{~h})$ & 862 & 39.3 & 1267 & 38.0 & \\
\hline $\operatorname{High}(>40 \mathrm{~h})$ & 626 & 28.5 & 1042 & 31.3 & \\
\hline Weekly TV viewing & & & & & $<0.0001$ \\
\hline Low $(<6 \mathrm{~h})$ & 767 & 34.9 & 858 & 25.7 & \\
\hline Moderate (6-15 h) & 963 & 43.9 & 1506 & 45.2 & \\
\hline $\operatorname{High}(>15 \mathrm{~h})$ & 465 & 21.1 & 968 & 29.1 & \\
\hline \multicolumn{6}{|l|}{ Elevated cardiometabolic risk } \\
\hline Waist circumference ${ }^{a}$ & 1130 & 51.5 & 1610 & 48.3 & 0.021 \\
\hline Dyslipidaemia $^{\mathrm{b}}$ & 509 & 23.2 & 1784 & 53.4 & $<0.0001$ \\
\hline Blood pressure ${ }^{c}$ & 735 & 33.5 & 2288 & 68.7 & $<0.0001$ \\
\hline Blood glucose ${ }^{\mathrm{d}}$ & 963 & 43.9 & 1280 & 38.4 & $<0.0001$ \\
\hline Inflammation $(\mathrm{CRP})^{\mathrm{e}}$ & 357 & 16.3 & 355 & 10.7 & $<0.0001$ \\
\hline No. cardiometabolic risk markers & & & & & $<0.0001$ \\
\hline Nil & 395 & 18.0 & 302 & 9.1 & \\
\hline One & 698 & 31.8 & 755 & 22.7 & \\
\hline Two & 556 & 25.3 & 894 & 26.8 & \\
\hline Three & 347 & 15.8 & 830 & 24.9 & \\
\hline Four & 152 & 6.9 & 471 & 14.1 & \\
\hline Five & 47 & 2.1 & 80 & 2.4 & \\
\hline Body mass index & & & & & $<0.0001$ \\
\hline Healthy $\left(<25 \mathrm{~kg} / \mathrm{m}^{2}\right)$ & 1110 & 50.6 & 721 & 21.6 & \\
\hline Over weight $\left(25-30 \mathrm{~kg} / \mathrm{m}^{2}\right)$ & 752 & 34.3 & 1845 & 55.4 & \\
\hline Obese $\left(>30 \mathrm{~kg} / \mathrm{m}^{2}\right)$ & 333 & 15.2 & 766 & 23.0 & \\
\hline
\end{tabular}

$S D$ standard deviation, GCSE general certificate of Secondary Education, EI energy intake

*Student $t$ test compared mean values between male and female participants. Chi-squared test compared differences between men and women for categorical variables, missing data was not included in the analyses, overall $\mathrm{p}$ value for Chi-square test presented. $\dagger$ METS metabolic equivalents, classification by IPAQ guidelines (21)

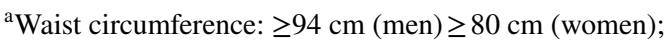

${ }^{\mathrm{b}}$ Dyslipidaemia HDL: $<1.0 \mathrm{mmol} / \mathrm{L}$ (men); $<1.3 \mathrm{mmol} / \mathrm{L}$ (women) and/or Non HDL $\geq 4 \mathrm{mmol} / \mathrm{L}$ and/or Reported diagnosed dyslipidaemia and/or self-reported lipid lowering medication

${ }^{\mathrm{c}}$ Blood pressure Systolic $\geq 130 \mathrm{mmHg}$ and/or diastolic $\geq 85 \mathrm{mmHg}$ and/or self-reported diagnosed hypertension and/or taking hypotensive medication

${ }^{\mathrm{d}}$ Blood glucose $\mathrm{HbA} 1 \mathrm{c} \geq 5.7 \%$ and/or self-reported diagnosed diabetes and/or taking medication for glucose control

${ }^{\mathrm{e}}$ Inflammation C-reactive protein $\geq 3 \mathrm{mg} / \mathrm{L}<10 \mathrm{mg} / \mathrm{L}, 39$ participants had missing or CRP values above $10 \mathrm{mg} / \mathrm{L}$ 
Table 2 Dietary intakes of men and women in the Airwave Health Monitoring Study ( $n$ 5527)

\begin{tabular}{|c|c|c|c|c|c|}
\hline \multirow[b]{2}{*}{ DASH score, median (range) } & \multicolumn{2}{|c|}{ Women $(n=2352)$} & \multicolumn{2}{|c|}{$\operatorname{Men}(n=3497)$} & \\
\hline & 24 & $9-38$ & 24 & $9-39$ & \\
\hline & \multicolumn{4}{|c|}{ Mean, SD } & $P$ \\
\hline Mean daily energy intake, kcal & 1674 & 386 & 2077 & 473 & $<0.0001$ \\
\hline Energy density food $\mathrm{kcal} / \mathrm{g}$ & 1.5 & 0.4 & 1.6 & 0.4 & $<0.0001$ \\
\hline$\%$ energy intake: total fat & 33.8 & 5.7 & 33.5 & 5.4 & 0.08 \\
\hline$\%$ energy intake: saturated fat & 12.4 & 2.9 & 12.3 & 2.8 & 0.67 \\
\hline$\%$ energy intake: protein & 17.0 & 3.4 & 17.2 & 3.4 & 0.028 \\
\hline$\%$ energy intake: carbohydrate & 48.0 & 7.1 & 46.7 & 6.9 & $<0.0001$ \\
\hline \multirow[t]{2}{*}{$\%$ energy intake NMEs } & 12.3 & 5.2 & 11.6 & 4.9 & $<0.0001$ \\
\hline & \multicolumn{5}{|c|}{ Median, IQR } \\
\hline$\%$ energy intake: alcohol $^{\mathrm{a}}$ & 2.9 & 6.6 & 4.4 & 7.6 & $<0.0001$ \\
\hline NSP g/1000 kcal & 7.1 & 2.9 & 6.6 & 2.7 & $<0.0001$ \\
\hline \multicolumn{6}{|l|}{ DASH score food group intakes } \\
\hline Fruit g/1000 kcal & 77.7 & 95.5 & 65.7 & 85.6 & $<0.0001$ \\
\hline Vegetables g/1000 kcal & 79.7 & 57.5 & 59.3 & 43.2 & $<0.0001$ \\
\hline Legumes g/1000 kcal & 11.7 & 15.4 & 10.9 & 13.0 & 0.031 \\
\hline Whole grains $\mathrm{g} / 1000 \mathrm{kcal}$ & 19.4 & 28.1 & 19.0 & 32.0 & 0.79 \\
\hline Low fat dairy $\mathrm{g} / 1000 \mathrm{kcal}$ & 96.6 & 91.8 & 92.4 & 83.3 & 0.008 \\
\hline Red meat g/1000 kcal & 49.5 & 46.7 & 74.1 & 54.4 & $<0.0001$ \\
\hline Sugar sweetened beverages $\mathrm{g} / 1000 \mathrm{kcal}$ & 34.7 & 110.9 & 29.4 & 103.0 & 0.017 \\
\hline \multirow[t]{2}{*}{ Sodium mg/1000 kcal/day } & 1449 & 407 & 14,60 & 373 & 0.24 \\
\hline & \multicolumn{5}{|l|}{$N, \%$} \\
\hline Discretionary salt usage $^{\mathrm{b}}$ & & & & & 0.19 \\
\hline No salt added & 999 & 52.9 & 1407 & 51.2 & \\
\hline Salt added either table or cooking & 506 & 26.8 & 742 & 27.0 & \\
\hline Salt added both table and cooking & 229 & 12.1 & 390 & 14.2 & \\
\hline Salt substitute used & 155 & 8.2 & 208 & 7.6 & \\
\hline
\end{tabular}

Student $t$ test compared mean values between male and female participants, Mann-Whitney $U$ test compared median values between male and female participants and Chi-squared test to compare differences between men and women across categorical variables

$I Q R$ interquartile range, NME non-milk extrinsic sugars, NSP non-starch polysaccharides (estimated from Englyst values).

${ }^{a}$ Includes non-consumers

${ }^{\mathrm{b}}$ Salt questionnaire completed by 4636 (missing data not included in analyses)

adjustment for established predictors of diet quality (age, education, household income, marital status, geographical region, smoking and TV viewing). Advancing age was associated with reduced odds of having a poor diet as was being in the highest category for physical activity. The highest category of education (bachelor degree or higher) was negatively associated with poor diet quality, and remained significant after adjustment (bachelor degree or higher vs. GCSE or below: men: OR $0.5395 \%$ CI $0.41,0.70$; women: OR $0.4395 \%$ CI $0.31,0.60$ ). For men and women geographical region and smoking status were positively associated with poor diet quality (Scotland vs. England: men: OR 1.88 95\% CI 1.53, 2.32; women: OR 1.49 95\% CI 1.11, 2.00; current vs. never smoker men: OR
$1.9095 \%$ CI 1.41, 2.58, women: OR 3.35 95\% CI 2.47, 4.55), Table 4. These associations remained significant after adjustment for established predictors of diet quality. High and passive job strain (vs. low) was associated with increased odds of having a poor diet for men, though after adjustment only high job strain reminded significant (OR $1.3895 \%$ CI $1.06,1.58)$. Working $49 \mathrm{~h}$ or more per week (compared to $<40 \mathrm{~h}$ ) was associated with poorer diet quality in men and women (men OR 1.53 95\% CI 1.21, 1.92; women: OR $1.5395 \%$ CI 1.12, 2.09), this only remained significant for men after adjustment. For men, the trend across ascending groups of working hours and increased cardiometabolic risk was significant $\left(P_{\text {trend }}=0.037\right)$. 
Table 3 Odds ratio of having three or more markers of metabolic risk per quintile of DASH score: $\operatorname{men}(n=3278)$ and women $(n=2139)$

\begin{tabular}{|c|c|c|c|c|c|c|c|}
\hline & \multirow[t]{2}{*}{ Cases/N } & \multicolumn{2}{|c|}{ Minimal adjusted $^{\mathrm{b}}$} & \multicolumn{2}{|c|}{ Fully adjusted $^{\mathrm{a}}$} & \multicolumn{2}{|c|}{$\begin{array}{l}\text { Fully adjusted }^{\mathrm{a}}+ \\
\text { BMI }\end{array}$} \\
\hline & & OR & $(95 \% \mathrm{CI})$ & OR & $(95 \% \mathrm{CI})$ & OR & $(95 \% \mathrm{CI})$ \\
\hline \multicolumn{8}{|l|}{ Men } \\
\hline Ref: Q5 (healthiest) & $235 / 636$ & 1.00 & & 1.00 & & 1.00 & \\
\hline Q4 & $265 / 658$ & 1.24 & $(0.98,1.57)$ & 1.19 & $(0.93,1.50)$ & 1.10 & $(0.84,1.44)$ \\
\hline Q3 & $330 / 763$ & 1.62 & $(1.29,2.03)$ & 1.54 & $(1.22,1.92)$ & 1.32 & $(1.02,1.71)$ \\
\hline Q2 & $271 / 640$ & 1.72 & $(1.36,2.19)$ & 1.52 & $(1.19,1.94)$ & 1.22 & $(0.92,1.60)$ \\
\hline Q1 (unhealthiest) & $256 / 581$ & 2.16 & $(1.67,2.77)$ & 1.84 & $(1.43,2.38)$ & 1.50 & $(1.12,2.00)$ \\
\hline$p$ trend & & $<0.0001$ & & $<0.0001$ & & 0.006 & \\
\hline \multicolumn{8}{|l|}{ Women } \\
\hline Ref: Q5 (healthiest) & $87 / 406$ & 1.00 & & 1.00 & & 1.00 & \\
\hline Q4 & $107 / 444$ & 1.39 & $(0.98,1.95)$ & 1.35 & $(0.92,1.92)$ & 1.35 & $(0.91,2.02)$ \\
\hline Q3 & $126 / 473$ & 1.85 & $(1.32,2.60)$ & 1.82 & $(1.29,2.58)$ & 1.74 & $(1.17,2.57)$ \\
\hline Q2 & $108 / 438$ & 1.84 & $(1.30,2.61)$ & 1.72 & $(1.21,2.48)$ & 1.57 & $(1.04,2.35)$ \\
\hline Q1 (unhealthiest) & $103 / 378$ & 2.21 & $(1.53,3.19)$ & 1.92 & $(1.35,2.84)$ & 1.84 & $(1.19,2.97)$ \\
\hline$p$ trend & & $<0.0001$ & & 0.0003 & & 0.005 & \\
\hline
\end{tabular}

$B M I$ body mass index, $C I$ confidence intervals, $O R$ odds ratio

24 participants did not have Hs-CRP available for metabolic risk calculation. Total cases $=1357 / 3278$ 15 participants did not have Hs-CRP available for metabolic risk calculation. Total cases =531/2139

${ }^{a}$ Fully adjusted: age + physical activity, smoking status, education and TV viewing, job strain, continuous variables: mean energy intake and mean alcohol g/day. Women additionally adjusted for menopause status

${ }^{\mathrm{b}}$ Minimal adjusted $=$ age at health screen

\section{Discussion}

The present study demonstrated that diet quality determined by DASH score to be negatively associated with cardiometabolic risk within British police force employees. Employees in the lowest fifth of DASH score distribution had increased odds of cardiometabolic risk independent of other lifestyle factors and BMI. This study has also identified that a poor dietary score was also associated with other negative lifestyle behaviours (inactivity, smoking and TV viewing). Furthermore, employees reporting long working hours and high job strain (for men) had increased odds of reporting a dietary intake that was associated with cardiometabolic risk.

Previous studies in German and US police officers have observed the prevalence of metabolic syndrome to be higher in this occupational group compared to the general population [24, 25]. In the present study, two-fifths of male police force employees had three or more cardiometabolic risk factors. This is higher than the estimated prevalence of metabolic syndrome for the general European population of $\sim 20-30 \%$ [26]. However, the prevalence amongst women in the present study was comparable to those reported in the general European population. The difference in the prevalence of increased metabolic risk may be due to the criteria applied in the present study (excluding triglycerides as unavailable and inclusion of CRP). Although the cardiometabolic scoring system used in the present study has not been validated to predict future cardiometabolic disease, it contains markers of risk (non-HDL and Hs-CRP) previously independently associated with cardiometabolic risk, and therefore, addresses the limitations of the metabolic syndrome definition previously cited by The CardioMetabolic Health Alliance [27].

There were clear dietary differences across men and women, with female police employees more likely to report a diet with a higher concentration of fruit and vegetables, and lower concentration of processed and red meat. This observation supports a previous study that also reported that women more likely make healthier choices (higher fruit and vegetable intake with lower intake of high fat foods) compared to men [28]. The differences in dietary intakes between men and women are likely to be multifactorial [28], but within the Airwave Health Monitoring Study cohort may relate to shorter working hours or different job roles allowing female employees more control over dietary choices.

Despite DASH being commonly applied to US cohorts and its recommendation by the American Heart Association, to date the DASH score has not been widely applied to UK cohorts to determine cardiometabolic health [11]. In the present study, classification of reporting a poorer quality dietary pattern (being in the lowest fifth for DASH score) was characterised by a total fat, saturated fat and NME intake above UK dietary guidelines, high intakes of alcohol, and less than two portions of fruit and vegetables per day (Supplementary 


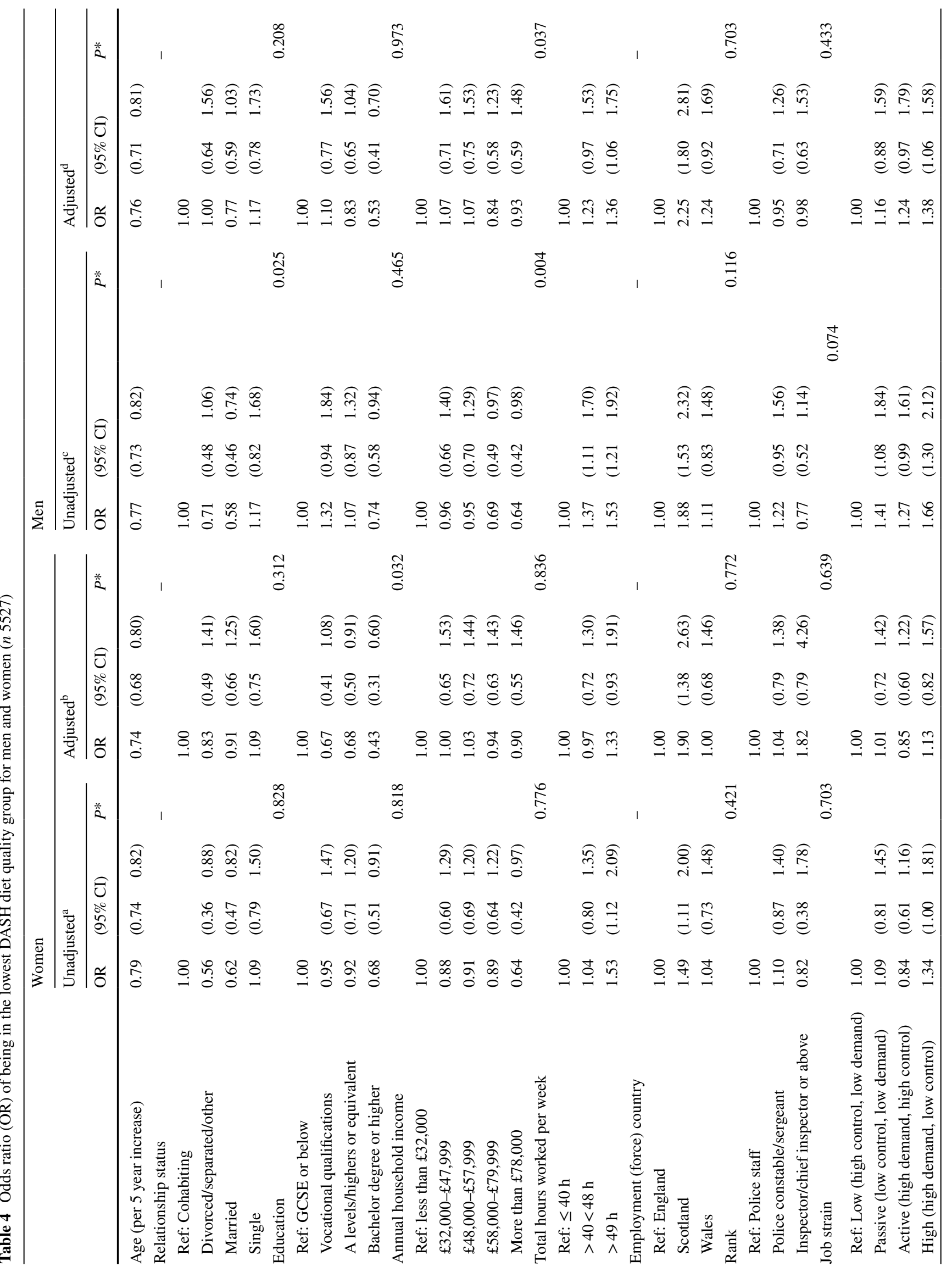




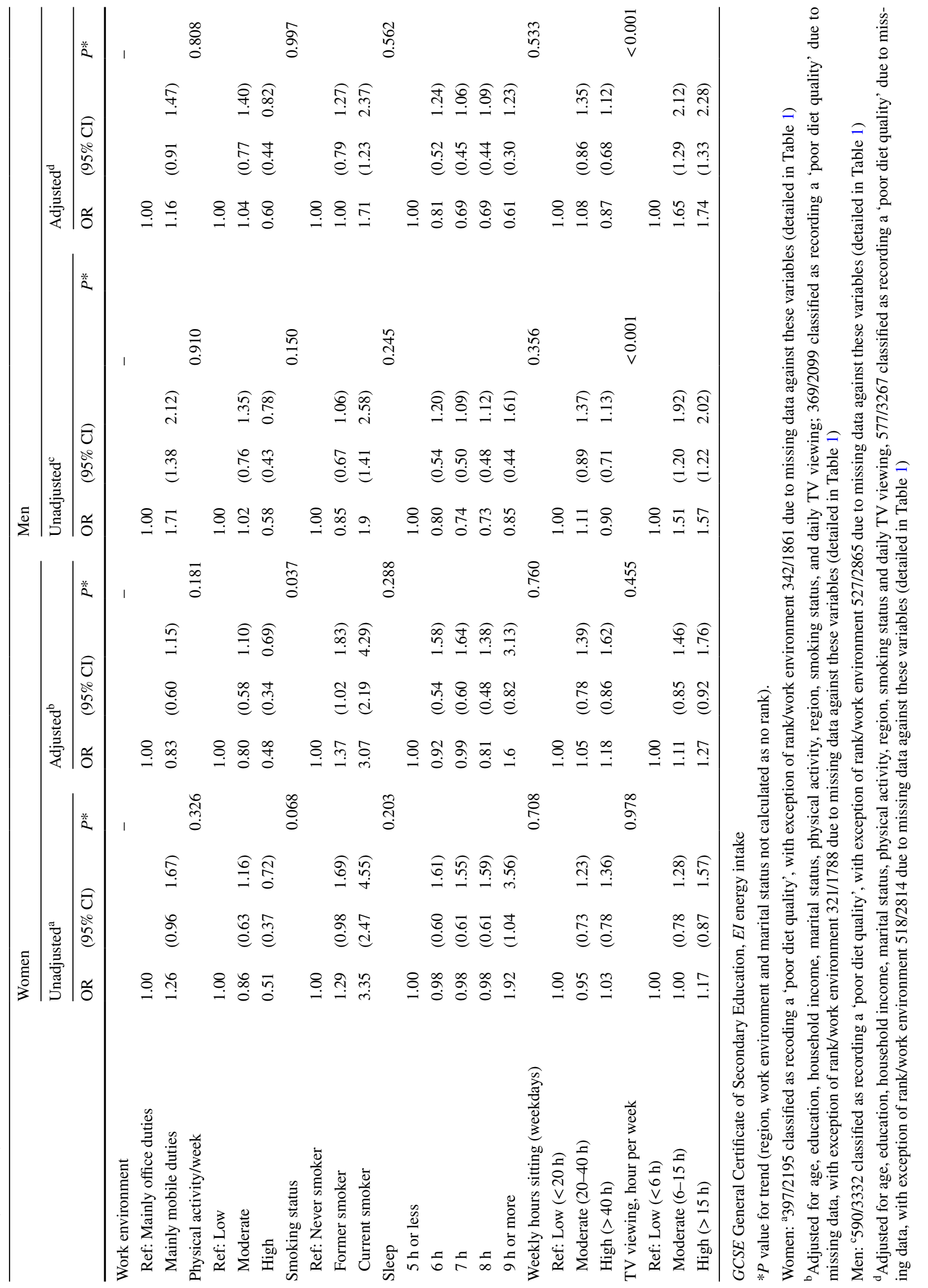


Table S3). The present study found that those in the lowest quintile of DASH score had over double the odds of having three or more metabolic risk markers compared to those in the highest DASH score category (age adjusted). The relationship across DASH score groups showed a dose-response with only slight attenuation in the strength of association after adjusting for other lifestyle factors (smoking, physical activity, TV viewing, energy intake) and BMI. The observation that DASH score was negatively associated with having three or more cardiometabolic risk markers is in agreement with a previous study in Iranian nurses that found participants in the highest DASH score tertile had $81 \%$ lower odds of metabolic syndrome [29]. In conjunction with a recent trial in the UK conferring the DASH diet adaptability and acceptability to a UK population [30] our observations suggest that the DASH diet could provide an effective intervention to improve cardiometabolic risk profile of police force employees and potentially the general UK population.

In agreement with previous studies in European populations, we observed advancing age [31] and higher education [32] to be negatively associated with poorer quality dietary intakes. We found no association between police rank and recording a poor quality diet. This finding does not reflect observations from the Whitehall II Study that reported employment grade to be associated with differences in dietary behaviours, with those in a lower employment grade consuming a poorer diet [33]. A possible explanation is that, within police employment ranks there is more heterogeneity in terms of job role and working environment compared to predominantly office-based civil servants. The geographical differences in dietary intakes were observed across regions of employment in the Airwave Health Monitoring Study largely reflect those previously reported [34]. Comprehensive analyses of British dietary intakes have identified populations in Scotland and Wales as consuming diets higher in saturated fat and sodium, and lower in fruit and vegetables compared to the English population [34]. The association between low DASH score and low physical activity and current smoking status are suggestive of an overall clustering unhealthy lifestyle behaviours, which have been reported previously [35]. A small cross-sectional study in police officers in Pennsylvania, USA $(n=247)$ observed that fruit and vegetable intake was positively associated with physical activity [36]. In the present study TV viewing, but not weekday sitting, was associated with a poor diet quality in men. The Nurses' Health Study (female only) similarly found that higher weekly TV viewing was associated with a diet high in energy, saturated fats, snacks and sweets [37].

We observed that the number of weekly working hours had a positive association with odds of recording a poor quality diet a relationship that was independent of age, education and region of employment for men. Previous studies investigating dietary intake and number of working hours have mainly used food frequency questionnaires or food surveys to collect dietary data [38-40], therefore, it is difficult to directly compare the results from the Airwave Health Monitoring Study. In general, our findings support the existing research that shows employees working longer weekly hours have higher intakes of take-away foods, convenience foods and snacks [38-40], which are indicative of a poorer quality diet. Job strain in men was also associated with diet quality, independent of established predictors. Previous research has shown psychological job demands to be positively associated with high fat food intakes in men, but not women [41]. High job strain is associated with longer working hours in the Airwave Health Monitoring Study Cohort. To determine if job strain contributed to the association between long hours and poorer diet quality in men exploratory analyses were conducted that repeated the multivariable logistic regression with both job strain and working hours (data not presented). Following this additional adjustment, long working hours ( $\geq 49 \mathrm{~h}$ per week) remained significantly associated with recording a poorer quality diet.

The strengths of the present study are the large-scale collection of 7-day estimated weighted diet records from a young single occupational group who are potentially at higher risk of cardiometabolic disease compared to the general population [24, 25]. The benefit of prospective measurement methods such as diet diaries, compared to food frequency questionnaires is that they do not measure against a predefined food list and allow more detailed dietary intake to be captured within occupational cohorts [42]. Additionally, food diaries do not rely on participant memory and recall ability. Additionally, the application of the DASH score to a UK population has been previously limited [11], therefore, these results strengthen the case for exploring the application of DASH diet guidelines as an intervention in the UK population. Our study has a number of limitations. Limited self-reported shift work information is currently only available for $12 \%$ of the cohort, and therefore, not included in the present study. The Buffalo Cardio-Metabolic Occupational Police Stress Study did observe an association between shift work and poor diet quality (measured by Dietary Inflammatory Index score) [43]. In the Airwave Health Monitoring Study cohort preliminary analyses have shown shift work prevalence is significantly associated with duration of weekly working hours (i.e., employees who are in the highest groups of weekly working hours are more likely to undertake shift work). Unfortunately, the interaction between shift work and long-working hours on determining dietary quality could not be explored due to the small sample size available. As with all current dietary recording tools, bias in reporting is an acknowledged limitation in nutritional research. Based on the food diary data, the estimated prevalence of misreporting energy intake was 50\% in the Airwave Health Monitoring Study, a figure that is comparable to that reported in 
studies of the general UK population [17]. We, therefore, conducted sensitivity analyses excluding those classed as under-reporting energy intake (data not presented). From these analyses, we observed the significant association between diet quality and high cardiometabolic risk was lost for men after adjustment for BMI, a finding that would be expected given that BMI is strongly associated with underreporting in the Airwave Health Monitoring Study Cohort [17]. The cross-sectional design of this study cannot infer a temporal pathway between long work hours or job strain and deterioration in dietary quality. Our observations may also be subject to residual confounding, as information on existing work place health initiatives was not collected as part of the study, this may be important to ascertain in future studies given that the Food Choice at Work Study in Ireland found employees with higher nutritional knowledge had a higher DASH score [44].

In conclusion, this study has profiled the dietary intakes and measured the diet quality (using the DASH score) of British police force employees. The novel aspect to this study has been the application of 7-day food diaries in the investigation of occupational exposures and diet quality. In general, the observed differences in diet quality across different employee characteristics (sex, region and age) in the police force reflect those within the general British population. Our findings suggest that for male police force employees longer duration of weekly working hours and higher job strain are associated with reporting a diet quality indicative of increased cardiometabolic risk. These findings potentially support the suggestion that dietary differences may contribute to variation in cardiometabolic disease risk observed across employees working longer hours or with high job strain. To test this hypothesis, future longitudinal studies are required to investigate if diet quality meditates the relationship between these occupational exposures and cardiometabolic disease. Additionally, further investigation to examine the barriers to healthy eating amongst male employees working extended hours or with high job strain is warranted. Gaining an understanding of why this cohort of employees are more likely to consume a diet associated with increased cardiometabolic risk will assist nutritional and occupational practitioners in targeting workplace nutritional interventions.

Acknowledgements We thank all participants in the Airwave Health Monitoring Study. We would also like to thank all the dietary coders who have contributed to the generation of the dietary data: Jessica Ayling, Andrea Carames, Zhengyu Fan, Kirsty Frost, Louise Hirichi, Zanna Hofstede, Kathryn Lamb, Yvonne McMeel, Niamh O'Sullivan, Kristina Petersen, Claudia Schreuder, Manny Singh, Elizabeth Slack, Jill Twomey, Alan Wan, Jessica Ware and Yiling Zhu. We also thank Louisa Cavaliero and Jeanette Spear who assisted in data collection and management.
Author contributions GF, PE, RG, formulated the research question and methodological design; RG was responsible for data analysis and drafting of the manuscript. GF, PE, A-CV, QC, RE, and RG, contributed to the interpretation of results and final manuscript. RG and $\mathrm{RE}$ managed the generation of the dietary data. A-CV supervised the creation of the non-dietary data extracts. DS was responsible for setting up the enrolment in each force and training clinic staff. AH developed and maintained the database. PE is the principal investigator of the Airwave Health Monitoring Study. All authors read and approved the final manuscript.

\section{Compliance with ethical standards}

Financial support The Airwave Health Monitoring Study is funded by the Home Office (grant number 780- TETRA) with additional support from the National Institute for Health Research (NIHR). The diet coding was supported through discretionary departmental funds. This paper presents independent research funded by the Home Office with additional support from the NIHR, ICHNT and Imperial College Biomedical Research Centre (BRC) at Imperial College Healthcare NHS Trust. P.E. and G.F. are NIHR senior investigators. P.E. acknowledges support from the NIHR Biomedical Research Centre at Imperial College Healthcare NHS Trust and Imperial College London, the NIHR Health Protection Research Unit on Health Effects of Environmental Hazards (HPRU-2012-10141), and the Medical Research Council-Public Health England (MRC-PHE) Centre for Environment and Health (MR/L01341X/1). PE is a UK Dementia Research Institute (DRI) Professor, UK DRI at Imperial College London. The UK DRI is funded by the Medical Research Council, Alzheimer's Society and Alzheimer's Research UK. This work used computing resources of the UK MEDical BIOinformatics partnership - aggregation, integration, visualisation and analysis of large, complex data (UK MED-BIO) which is supported by the Medical Research Council (grant number MR/L01632X/1). The Section of Endocrinology and Investigative Medicine is funded by grants from the MRC, Biotechnology and Biological Sciences Research Council (BBSRC), NIHR, an Integrative Mammalian Biology (IMB) Capacity Building Award, an FP7-HEALTH-2009-241592 EuroCHIP grant and is supported by the NIHR Biomedical Research Centre Funding Scheme. The views expressed are those of the authors and not necessarily those of the Home Office, ICHNT, the BRC, the NHS, the NIHR or the Department of Health.

Financial support The Airwave Health Monitoring Study is funded by the Home Office (grant number 780- TETRA) with additional support from the National Institute for Health Research (NIHR). The diet coding was supported through discretionary departmental funds. This paper presents independent research funded by the Home Office with additional support from the NIHR, ICHNT and Imperial College Biomedical Research Centre (BRC) at Imperial College Healthcare NHS Trust. P.E. and G.F. are NIHR senior investigators. P.E. acknowledges support from the NIHR Biomedical Research Centre at Imperial College Healthcare NHS Trust and Imperial College London, the NIHR Health Protection Research Unit on Health Effects of Environmental Hazards (HPRU-2012-10141), and the Medical Research Council-Public Health England (MRC-PHE) Centre for Environment and Health (MR/L01341X/1). PE is a UK Dementia Research Institute (DRI) Professor, UK DRI at Imperial College London. The UK DRI is funded by the Medical Research Council, Alzheimer's Society and Alzheimer's Research UK. This work used computing resources of the UK MEDical BIOinformatics partnership - aggregation, integration, visualisation and analysis of large, complex data (UK MED-BIO) which is supported by the Medical Research Council (grant number MR/L01632X/1). The Section of Endocrinology and Investigative Medicine is funded by grants from the MRC, Biotechnology and Biological Sciences Research Council (BBSRC), NIHR, an Integrative Mammalian Biology (IMB) 
Capacity Building Award, an FP7-HEALTH-2009-241592 EuroCHIP grant and is supported by the NIHR Biomedical Research Centre Funding Scheme. The views expressed are those of the authors and not necessarily those of the Home Office, ICHNT, the BRC, the NHS, the NIHR or the Department of Health.

Conflict of interest On behalf of all authors, the corresponding author states that there is no conflict of interest.

Ethical approval The Airwave Health Monitoring Study is approved by the National Health Service Multi-site Research Ethics Committee (MREC/13/NW/0588).

Informed consent All subjects gave their informed consent prior to their inclusion in the study.

Open Access This article is distributed under the terms of the Creative Commons Attribution 4.0 International License (http://creativecommons.org/licenses/by/4.0/), which permits unrestricted use, distribution, and reproduction in any medium, provided you give appropriate credit to the original author(s) and the source, provide a link to the Creative Commons license, and indicate if changes were made.

\section{References}

1. World Health Organization (1985) Identification and control of work-related diseases. Geneva, WHO

2. Gan Y, Yang C, Tong X, Sun H, Cong Y, Yin X et al (2014) Shift work and diabetes mellitus: a meta-analysis of observational studies. Occup Environ Med 72(1):72-78

3. Kivimäki M, Jokela M, Nyberg ST, Singh-Manoux A, Fransson EI, Alfredsson L et al (2015) Long working hours and risk of coronary heart disease and stroke: a systematic review and metaanalysis of published and unpublished data for 603838 individuals. Lancet 386(10005):1739-1746

4. Chandola T, Brunner E, Marmot M (2006) Chronic stress at work and the metabolic syndrome: prospective study. BMJ 332(7540):521-525

5. Department of Health (2014) NHS Choices Boost your health at work. http://www.nhs.uk/Livewell/workplacehealth/Pages/workplaceoverview.aspx Accessed 5 Jan 2015

6. Ezzati M, Riboli E (2013) Behavioral and Dietary Risk Factors for Noncommunicable Diseases. N Engl J Med 369(10):954-964

7. Dyson PA, Kelly T, Deakin A, Duncan A, Frost G, Harrison Z et al (2011) Diabetes UK evidencebased nutrition guidelines for the prevention and management of diabetes. Diabet Med 28(11):1282-1288

8. National Institute for Health and Care Excellence (2015) Maintaining a healthy weight and preventing excess weight gain among adults and children. NICE guidelines NG7

9. Calton EK, James AP, Pannu PK, Soares MJ (2014) Certain dietary patterns are beneficial for the metabolic syndrome: reviewing the evidence. Nutr Res 34(7):559-568

10. de Koning L, Chiuve SE, Fung TT, Willett WC, Rimm EB, Hu FB (2011) Diet-quality scores and the risk of type 2 diabetes in men. Diabetes Care 34(5):1150-1156

11. Siervo M, Lara J, Chowdhury S, Ashor A, Oggioni C, Mathers JC (2015) Effects of the dietary approach to stop hypertension (DASH) diet on cardiovascular risk factors: a systematic review and meta-analysis. Br J Nutr 113(1):1-15
12. Fung TT, Chiuve SE, McCullough ML, Rexrode KM, Logroscino G, Hu FB (2008) Adherence to a DASH-style diet and risk of coronary heart disease and stroke in women. Arch Intern Med 168(7):713-720

13. Ball K, Timperio AF, Crawford DA (2006) Understanding environmental influences on nutrition and physical activity behaviors: where should we look and what should we count? Int J Behav Nutr Phys Act 3:33

14. Association of Chief Police Officers (2012) UK Police Directory 2012. Chichester: Keyways Publishing Ltd

15. Elliott P, Vergnaud A-C, Singh D, Neasham D, Spear J, Heard A et al (2014) The Airwave Health Monitoring Study of police officers and staff in Great Britain: Rationale, design and methods. Environ Res 134C:280-285

16. Nelson M, Haraldsdottir J (1998) Food photographs: practical guidelines: development and use of photographic atlases for assessing food portion size. Pub Health Nutr 1:231-237

17. Gibson R, Eriksen R, Lamb K, McMeel Y, Vergnaud AC, Spear J et al (2017) Dietary assessment of British police force employees: a description of diet record coding procedures and cross-sectional evaluation of dietary energy intake reporting (The Airwave Health Monitoring Study. BMJ Open 7(4):e012927

18. Black AE (2000). Critical evaluation of energy intake using the Goldberg cut-off for energy intake:basal metabolic rate. A practical guide to its calculation, use and limitations. Int J Obes Relat Metab Disord;24:1119-1130

19. Landsbergis PA, Schnall PL, Pickering TG, Schwartz JE (2002) Validity and reliability of a work history questionnaire derived from the Job Content Questionnaire. J Occup Environ Med 44(11):1037-1047

20. Ingre M (2015) Excuse me, but did the IPD-work consortium just "falsify" the job-strain model? Scand J Work Environ Health 1(5):504-505

21. Craig CL, Marshall AL, Sjöström M, Bauman AE, Booth ML, Ainsworth BE et al (2003) International physical activity questionnaire: 12-country reliability and validity. Med Sci Sports Exerc 35(8):1381-1395

22. IPAQ scoring protocol (2005) http://www.ipaq.ki.se/scoring.htm Accessed 20 Oct 2014

23. Beasley TM, Schumacker RE (1995) Multiple regression approach to analyzing contingency tables: Post hoc and planned comparison procedures. J Exp Educ 64(1):79-93

24. Anderson AA, Yoo H, Franke WD (2016) Associations of physical activity and obesity with the risk of developing the metabolic syndrome in law enforcement officers. J Occup Environ Med 58(9):946-951

25. Humbarger CD, Crouse SF, Womack JW, Green JS (2004) Frequency of metabolic syndrome in police officers compared to NCEP III prevalence values. Med Sci Sport Exerc 36(5):S161

26. Grundy SM (2008) Metabolic syndrome pandemic. Arterioscler Thromb Vasc Biol 28(4):629-636

27. Sperling LS, Mechanick JI, Neeland IJ, Herrick CJ, Després J-P, Ndumele CE et al (2015) The cardioMetabolic health alliance: working toward a new care model for the metabolic syndrome. J Am Coll Cardiol 66(9):1050-1067

28. Wardle J, Haase AM, Steptoe A, Nillapun M, Jonwutiwes K, Bellisie $\mathrm{F}$ (2004) Gender differences in food choice: the contribution of health beliefs and dieting. Ann Behav Med 27(2):107-116

29. Saneei P, Fallahi E, Barak F, Ghasemifard N, Keshteli AH, Yazdannik AR et al (2015) Adherence to the DASH diet and prevalence of the metabolic syndrome among Iranian women. Eur J Nutr 54(3):421-428

30. Harnden KE, Frayn KN, Hodson L (2010) Dietary approaches to stop hypertension (DASH) diet: applicability and acceptability to a UK population. J Hum Nutr Diet 23(1):3-10 
31. Alkerwi A, Vernier C, Crichton GE, Sauvageot N, Shivappa N, Hébert JR (2014) Cross-comparison of diet quality indices for predicting chronic disease risk: findings from the Observation of Cardiovascular Risk Factors in Luxembourg (ORISCAV-LUX) study. Br J Nutr 5(2):111-113(

32. Finger JD, Tylleskär T, Lampert T, Mensink GBM, Irala-Estevez J, De Groth M et al (2013) Dietary behaviour and socioeconomic position: the role of physical activity patterns. PLoS One Pub Library Sci 8(11):e78390

33. Marmot MG, Stansfeld S, Patel C, North F, Head J, White I et al (1991) Health inequalities among British civil servants: the Whitehall II study. Lancet 337(8754):1387-1393

34. Scarborough P, Morgan RD, Webster P, Rayner M (2011) Differences in coronary heart disease, stroke and cancer mortality rates between England, Wales, Scotland and Northern Ireland: the role of diet and nutrition. BMJ Open 1(1):e000263

35. Alkerwi A, Vernier C, Crichton GE, Sauvageot N, Shivappa N, Hébert JR (2014) Cross-comparison of diet quality indices for predicting chronic disease risk: findings from the Observation of Cardiovascular Risk Factors in Luxembourg (ORISCAV-LUX) study. Br J Nutr 113(2):259-269

36. Can SH, Hendy HM (2014) Behavioral variables associated with obesity in police officers. Ind Health 52(3):240-247

37. Hu FB, Li TY, Colditz GA, Willett WC, Manson JE (2003) Television watching and other sedentary behaviors in relation to risk of obesity and type 2 diabetes mellitus in women. JAMA 289(14):1785-1791

38. Fan W, Lam J, Moen P, Kelly E, King R, McHale S (2015) Constrained choices? Linking employees' and spouses' work time to health behaviors. Soc Sci Med 126:99-109

39. Escoto KH, Laska MN, Larson N, Neumark-Sztainer D, Hannan PJ (2012) Work hours and perceived time barriers to healthful eating among young adults. Am J Health Behav 36(6):786-796

40. Escoto KH, French SA, Harnack LJ, Toomey TL, Hannan PJ, Mitchell NR (2010) Work hours, weight status, and weight-related behaviors: a study of metro transit workers. Int J Behav Nutr Phys Act 7:91

41. Hellerstedt WL, Jeffery RW (1997) The association of job strain and health behaviours in men and women. Int $\mathrm{J}$ Epidemiol 26(3):575-583

42. Lindström J (2016) Does higher energy intake explain weight gain and increased metabolic risks among shift workers? Scand J Work Environ Health 1(6):455-457

43. Wirth MD, Burch J, Shivappa N, Violanti JM, Burchfiel CM, Fekedulegn D et al (2014) Association of a dietary inflammatory index with inflammatory indices and metabolic syndrome among police officers. J Occup Environ Med 56(9):986-989

44. Geaney F, Fitzgerald S, Harrington JM, Kelly C, Greiner BA, Perry IJ (2015) Nutrition knowledge, diet quality and hypertension in a working population. Prev Med Rep 2:105-113 